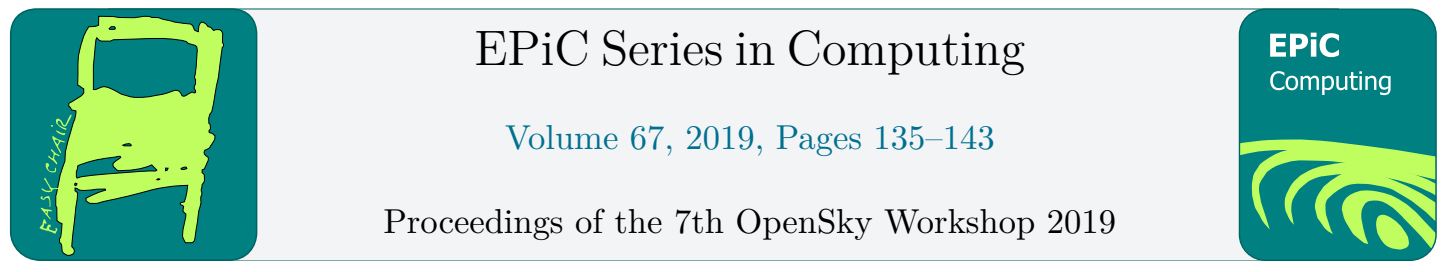

\title{
Investigating ADS-B MOPS Compliance using Open Data
}

\author{
Enrico Spinielli \\ EUROCONTROL, Performance Review Service \\ Brussels, Belgium \\ enrico.spinielli@eurocontrol.int
}

\begin{abstract}
The introduction of Automatic Dependent Surveillance-Broadcast (ADS-B) in Aviation as mandated in the US [3,4] and in Europe [1,2] rests on the (at least theoretical) benefits of the switch of paradigm in ATC surveillance from (continuously) active interrogation of aircraft positions by primary radars to (almost always) automatic broadcast of data by the aircraft. The cost/benefit analysis in favour of this shift weights on the 1) higher frequency and precision of the aircraft information (position, speed, ...) made available to ATC/neighbouring aircraft leading to increased safety and increased airspace capacity and 2) on the (at least one) order of magnitude reduction in ground infrastructure costs [5]. Infrastructure needs nontheless to be deployed both on the ground and in the air; and in the end it is the passenger who pays via taxes on tickets or airports services: so what is the status of deployment? This paper investigates, using only open and free data, the status of compliance of aircraft in the European airspace, i.e. how many aircraft flying in Europe comply to the EASA ADS-B mandate.
\end{abstract}

\section{Introduction}

The introdution of ADS-B in aviation is justified by the (at least theoretical) benefits of the switch of paradigm in ATC surveillance from (continuously) active interrogation of aircraft positions by primary radars to (almost always) automatic broadcast of position (et al.) data by the aircraft.

The cost/benefit analysis in favour of this shift weights on the

1. higher frequency and precision of the aircraft information (identification, position, ...) made available to ATC/neighbouring aircraft leading to increased safety and increased airspace capacity; and

2. on the (at least one) order of magnitude reduction in ground infrastructure costs [5].

The (not so anticipated) drastic reduction in hardware price, availability of open software defined radio (SDR) kits combined with cheap Internet connectivity have made it possible for aviation enthusiasts to crowdsource ADS-B out data via platforms of companies such as FlightRadar24 (FR24), FlightAware (FA) or non-profit organizations such as OpeSky Network 
(OSN), ADS-B Exchange (AEX). These entities (or new entrants operating from space, Aireon) generally complement the collected data and make it available freely or against a fee.

The costs associated with the deployment of ADS-B Link architecture are borne by Airspace Service Providers (ANSP) for ground infrastructure and Airlines for equipment in the aircraft. But ultimtely the ATC services and the cost of Aircraft compliance are paid by the air passengers through the en-route and airport taxes on their tickets.

The open ADS-B data provide citizens and customers an important source of information that can be used to keep in check service providers both public and private in order to verify safety, compliance and proper use of the finances devoted to the implementation of the ADS-B network.

This paper investigates the possibilities and difficulties in analysing the status of compliance to ADS-B mandate for airlines flying in Europe using only openly available data sources.

In the process we will investigate the possibile open data sources to:

- identify the aircraft type (i.e. its weight category), airline, country of registration, ...

- determine the ADS-B Minimum Operational Performance Specifications (MOPS) of the equipment on the aircraft

We will also suggest area where the aviation community could focus some of its efforts in order to make some of the analysis steps in this paper possible, easier and/or more precise and reliable.

\section{Method}

The Performance Review Unit (PRU) evaluates EUROCONTROL Members States performance both from a financial and an operational perspective. In the operational context PRU has developed performance indicators to assess the efficiency of operations in the en-route and in the terminal control area (TMA).

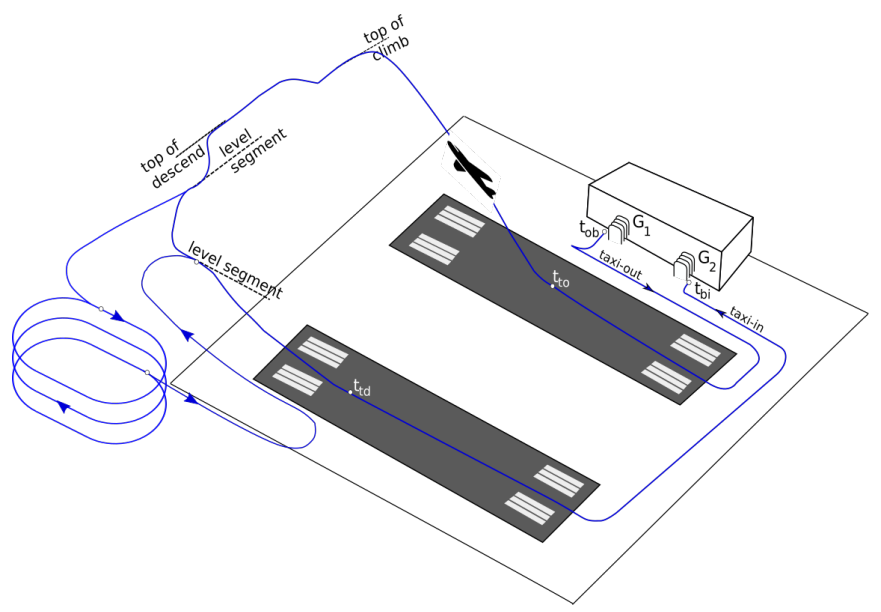

Figure 1: A simplified gate-to-gate flight trajectory.

Given that airports are more and more the constraining resources in the aviation network, the higher granularity and precision of ADS-B trajectories allow for the study of new aspects 
Table 1: Five major European airports.

\begin{tabular}{llrr}
\hline id & name & longitude & latitude \\
\hline EGLL & Heathrow Airport & -0.4613333 & 51.47750 \\
EDDF & Frankfurt am Main Airport & 8.5705000 & 50.03333 \\
LEMD & Adolfo Suárez Madrid-Barajas Airport & -3.5608333 & 40.47217 \\
LIRF & Rome-Fiumicino International Airport & 12.2388333 & 41.80033 \\
EHAM & Amsterdam Airport Schiphol & 4.7641667 & 52.30800 \\
\hline
\end{tabular}

of performance in the TMA such as Continuous Descend Operations (CDO), distance flown (and tonnes of $\mathrm{CO}_{2}$ produced) in holding stacks, ..., that are not possible with data currently available via the Network Manager.

Given the focus on the TMAs, for the scope of this paper 5 major European airports have been selected as described in Table 1 and mapped in Figure 2. For each of them one year of data points have been collected for the volume within an axis-aligned bounding box 40 nautical miles around the aiport reference points (ARP).

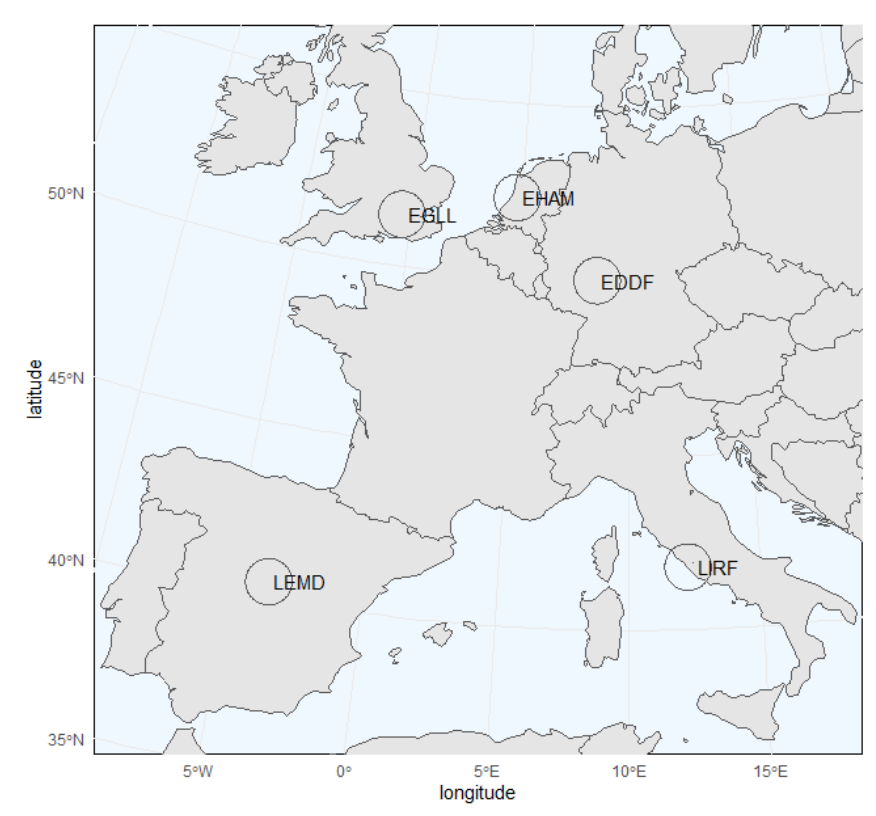

Figure 2: The 5 major airports and the 40 NM radius around their ARP.

The criteria for the selection of the airports is as follows:

1. they are major airports, so they have a reduced number of general aviation flights in their TMA and as such they will deal mainly with IFR flights, the subject of the EASA Regulation on ADS-B [1,2] 
2. they are of interest for PRU performance review;

3. they are geographically dispersed in Europe

Data collected in the TMA per hour is about

- minimalistic State Vector (msv) messages: all unique icao24, callsign pairs. This is a measure for the aircraft being present in the TMA;

- Operational Status messages (os): all unique icao24, rawmsg pairs for the above msv's.

The os's have been decoded using pyModes [9] in order to extract the relevant ADS-B version (either 1 or 2). The msv has been joined with os and the adsb_version column has been further filled forward and backward; finally the remaining empty versions have been assigned as 0 (see ADS-B Version section in [8].)

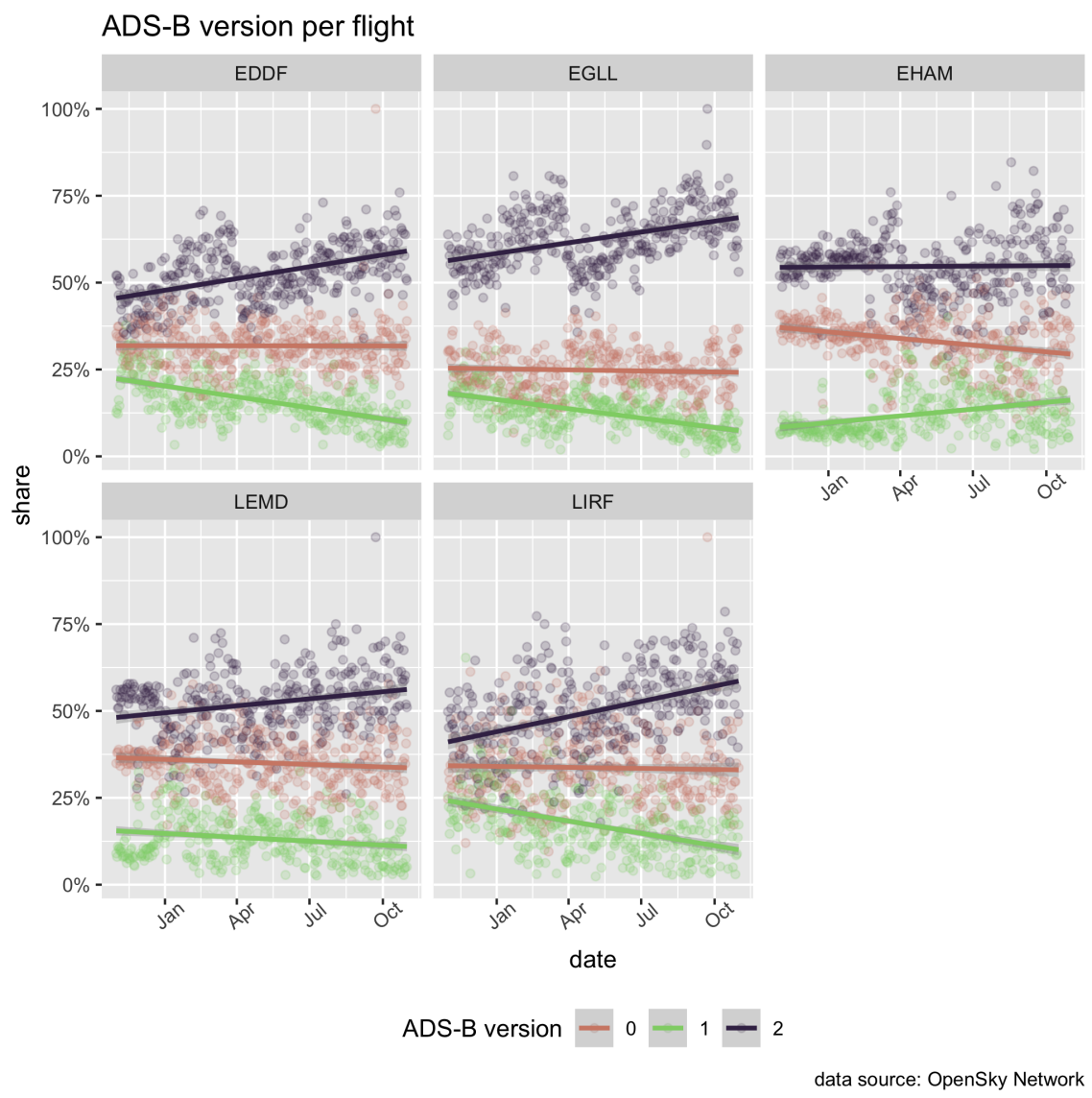

Figure 3: ADS-B version share per airport. 


\section{Results and Discussion}

\subsection{Flight and aircraft}

When we examine the ADS-B version trends per flight ${ }^{1}$ at each airport Figure 3 you can notice a general tendency towards larger and larger number of flights using ADS-B version 2.

A look at aircraft ${ }^{2}$ trend, Figure 4, confirms what shown at the airports level. The different trend compared to the airports one could be explained by the fact that version 0 aircraft operate more flights than version 2 ones, i.e. more regional/european flights compared to translatlantic flights being operated by aircraft having to be compliant to the FAA mandate.

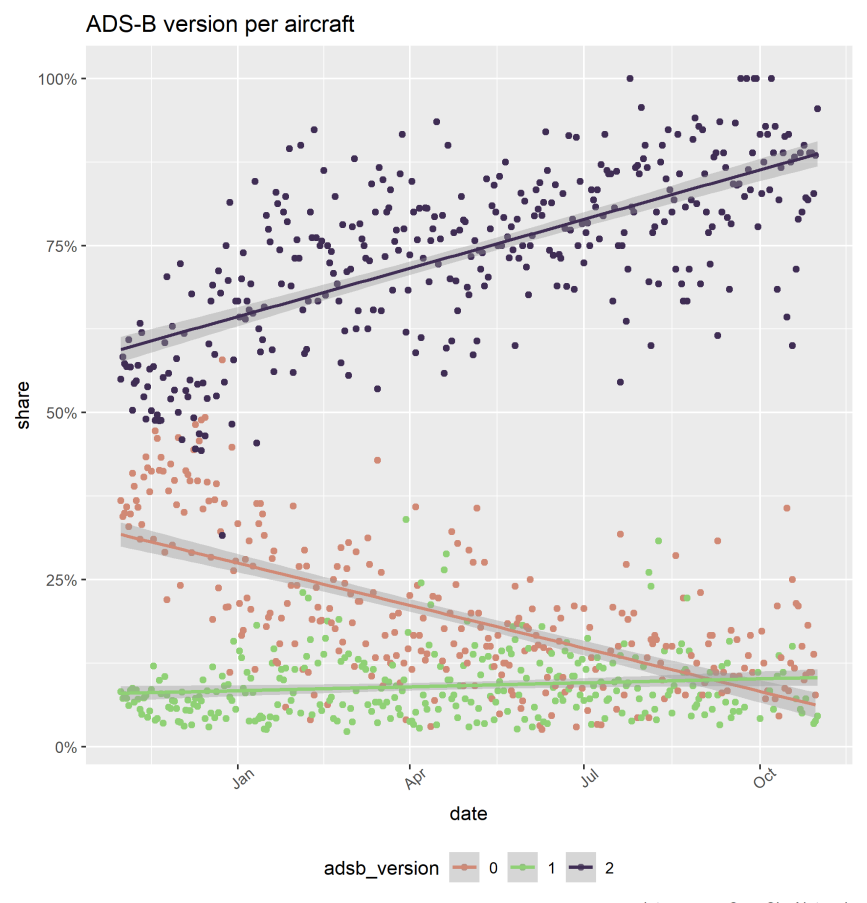

Figure 4: ADS-B version share per aircraft

The percentages in Figure 4 (Version 2 at $68 \%$ in Apr 2019) do not agreed with what claimed (40\%) on the SESAR Deplyment Manager website [7] but no details are provided to further investigate the differences.

\section{Country and aircraft type}

In order to analyse ADS-B version per aircraft type and/or aircraft operator we have joined the Aircraft DB from OSN but found the following shortcomings:

- $12 \%$ of the joined aircraft where missing country information. Extracting the registrar's

\footnotetext{
${ }^{1}$ in the analysis we have taken 1 flight $=$ unique pair (icao24, callsign)

${ }^{2}$ in the analysis we used 1 aircraft $=1$ unique icao24
} 
country from the ICAO block as per Table 9-1 of [6] left only 33 invalid/fake ones, see Table 2.

- $21 \%$ of aircraft missing registration

- the vast majority (88\%) missing operator

- while $96 \%$ of the aircraft DB has a model value, its encoding varies even within the same model, i.e. "A320 231", "Airbus A320 231", ..., "B1-RD", "B1-RD (Robertson)", "B1RD (INSTRUCTOR) (Robertson)", or "AERO AT-3 R100", "AT-3 R100", "AT-3", or "Airbus A320 214", "A320 214", "A320-214"

Table 2 for fake 24-bit addresses could be useful for authorities to spot and notify malfunctions/incorrect setups to aircraft operators.

The use of other aircraft/fleet databases, such as https://github.com/junzis/aircraft-db or https://planespotters.net, is limited by licenses and or commercial terms and as such do not fit the purpouse of this study to rely on free open data sources.

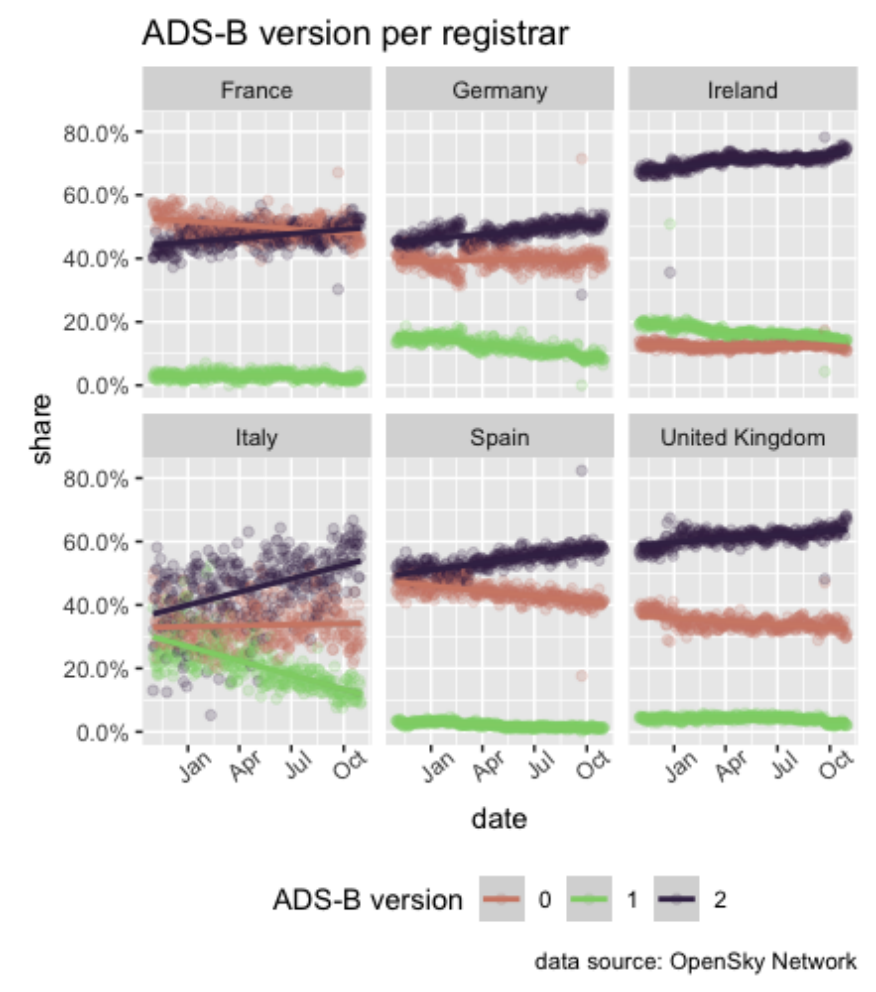

Figure 5: ADS-B version trend per (European) registrars' country

Nontheless the registrars' country can be devised via the block allocation as published by ICAO [6] and it can be used to plot the trend in ADS-B version over time as seem through the 5 sampling areas of this study.

Figure 5 shows a distinct high percentage of ADS-B version 2 aircraft in Ireland probably due to the relatively new fleet from RyanAir compared to more traditional airlenes from the other countries. The wide spread of data points in Italy is probably linked to the much smaller number of flights on LIRF resulting a wider variation of the counts. 


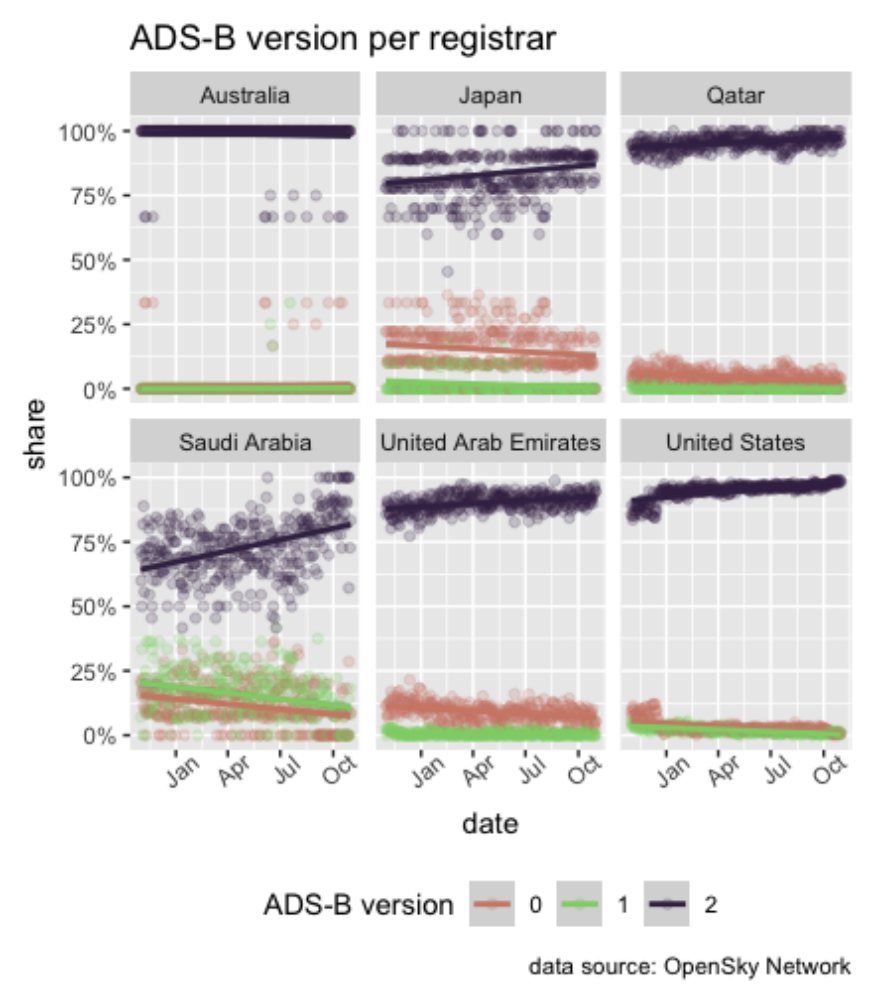

Figure 6: ADS-B version trend per (non-European) registrars' country

With respect to non European registrars Figure 6 shows a good level of adoption for USA due to the close deadline of Jan, 12020.

The plots for Japan, Australia and Saudi Arabia seem to suffer for low sample representation, so a wider sampling area is needed to draw conclusions.

\subsection{Future work}

There is the need for an up-to-date fleet database: it would be great to be able to create a community around a project to openly maintain a repository of this data. National aircraft registrars make some aircraft info available and could be a starting point. For example the Irish Aviation Authority (or the Swiss just to cite another excellent example) provides owner, aircraft type, operator and ICAO 24-bit addresses too, many others unfortunately limit the data to some of immatriculation, manifacturer, aircraft type, serial number, owner, operator and typically lack 24-bit addresses. Sadly registrars' web sites are in general mainly for human consumption and little is done to ease data exchange via API or by automated procedures.

\section{Conclusions}

The trend in ADS-B version compliance is going in the right direction but it looks very likely that many aircraft will not be compliant with the EU Directive by the deadline of June, 7th 2020 . 
It is shocking that no public official data is made available by the supervising authority on the status of compliance on the ground AND in the air. While FAA provide some summaries, the underlying datasets are not disclused [5]. The situation in Europe is much worse.

For an open, independent analysis of ADS-B adoption and maintenance a fleet database is of the utmost importance. It could be interesting to try to combine disparate efforts by different communities and enthusiasts ${ }^{3}$ in order to funnel energies in the same direction and for the benefits of all.

\section{References}

[1] European Commission. 2011. Commission Implementing Regulation (EU) No 1207/2011 of 22 November 2011 laying down requirements for the performance and the interoperability of surveillance for the single European sky. Retrieved from http://eur-lex.europa.eu/legalcontent/EN/TXT/?uri=CELEX:32011R1207

[2] European Commission. 2017. Commission Implementing Regulation (EU) 2017/386 of 6 March 2017 amending Implementing Regulation (EU) No 1207/2011 laying down requirements for the performance and the interoperability of surveillance for the single European sky. Retrieved from http://data.europa.eu/eli/reg_impl/2017/386/oj

[3] FAA. 2010. 14 CFR 91.225 - Automatic Dependent Surveillance-Broadcast (ADS-B) Out equipment and use. Retrieved from https://www.ecfr.gov/cgi-bin/text-idx?SID=8137158 693744ba666e318c1f474d81b\&node=se14.2.91_1225\&rgn=div8

[4] FAA. 2010. 14 CFR 91.227 Automatic Dependent Surveillance-Broadcast (ADS-B) Out equipment performance requirements.

[5] FAA. 2019. ADS-B Frequently Asked Questions (FAQs). Retrieved from https://www.faa. gov/nextgen/programs/adsb/faq/\#g1

[6] ICAO. 2007. Annex 10 - Aeronautical Telecommunications - Volume 3 (second ed.).

[7] SESAR Deployment Manager. 2019. EU ADS-B airborne equipage trend accelerating. ADS$B$. Retrieved from https://ads-b-europe.eu/eu-ads-b-airborne-equipage-trend-accelerating/

[8] Junzi Sun. 2017. The 1090MHz Riddle - The book about decoding Mode-S and ADS-B data. Retrieved from https://github.com/junzis/the-1090mhz-riddle

[9] J. Sun, H. Vû, J. Ellerbroek, and J. M. Hoekstra. 2019. pyModeS: Decoding mode-s surveillance data for open air transportation research. IEEE Transactions on Intelligent Transportation Systems (2019). DOI:https://doi.org/10.1109/TITS.2019.2914770

\footnotetext{
${ }^{3}$ the author contacted planespotters.net, planelist.net
} 
Table 2: Invalid ICAO 24-bit addresses.

\begin{tabular}{|c|c|c|c|c|}
\hline icao24 & callsign & airport & date & adsb_version \\
\hline 0ca56d & RYR4TD & EDDF & 2018-11-01 & 2 \\
\hline 000023 & PH4P4 & EHAM & 2018-11-01 & 2 \\
\hline cba9a9 & PLM301P & LEMD & 2018-11-01 & 0 \\
\hline cba9a8 & PLL320P & LEMD & 2018-11-01 & 0 \\
\hline 0cabb9 & RYR8YC & EDDF & 2018-11-02 & 0 \\
\hline 096770 & PHTOO & EHAM & 2018-11-02 & 2 \\
\hline cba9e7 & LQV960P & EDDF & 2018-11-04 & 2 \\
\hline 592000 & UAF802 & EDDF & 2018-11-05 & 2 \\
\hline 001100 & IWALK & EDDF & 2018-11-06 & 2 \\
\hline $6 \mathrm{~d} 0113$ & CLX97E & EGLL & 2018-11-23 & 2 \\
\hline 892354 & ETD140 & EGLL & 2018-11-28 & 0 \\
\hline 000001 & TARTN12 & EGLL & 2018-12-03 & 2 \\
\hline fa0001 & FAV2 & LIRF & 2018-12-07 & 0 \\
\hline 000400 & PROVA20 & LIRF & 2018-12-11 & 1 \\
\hline 999999 & FHJRX & LIRF & 2018-12-18 & 2 \\
\hline $0020 \mathrm{cc}$ & $\mathrm{NA}$ & EDDF & 2019-01-02 & 1 \\
\hline 4cea19 & RYR6FM & LEMD & 2019-01-03 & 0 \\
\hline 5cea19 & RYR6YW & LEMD & 2019-01-07 & 0 \\
\hline $5051 \mathrm{c} 7$ & ATG6616 & EDDF & 2019-01-18 & 0 \\
\hline 5 caa19 & RYR3ET & EGLL & 2019-01-22 & 0 \\
\hline 646032 & P2008 & LIRF & 2019-02-15 & 2 \\
\hline $04 \mathrm{~d} 3 \mathrm{c} 8$ & NATO06 & EHAM & 2019-02-26 & 2 \\
\hline $2 \mathrm{cf} 6 \mathrm{c} 2$ & ADN15A & EDDF & 2019-03-15 & 2 \\
\hline 4da058 & AS1126 & LIRF & 2019-04-02 & 1 \\
\hline 0e8faa & 5NMPB & EHAM & 2019-04-08 & 2 \\
\hline 200450 & GCCMR & EGLL & 2019-04-14 & 2 \\
\hline 4 ce 819 & RYR191B & LEMD & 2019-04-24 & 0 \\
\hline 000111 & G941 & EGLL & 2019-05-03 & 2 \\
\hline 4cea11 & RYR4CP & LIRF & 2019-05-24 & 0 \\
\hline 4da4ea & RYR2FY & EGLL & 2019-06-03 & 2 \\
\hline fac688 & NA & LIRF & 2019-06-20 & 0 \\
\hline 002091 & R20221 & EDDF & 2019-08-27 & 2 \\
\hline 5ca811 & RYR220D & EGLL & 2019-10-04 & 0 \\
\hline
\end{tabular}

